\title{
INTERFERÊNCIAS SOCIOCULTURAIS E INSTITUCIONAIS NO ACESSO DO HOMEM AOS SERVIÇOS DE ATENÇÃO PRIMÁRIA À SAÚDE
}

\author{
Carla Gonçalves AGUIAR ${ }^{1}$ \\ Linda Márcia Freitas CÂMARA ${ }^{2}$ \\ Jucimere Fagundes Durães ROCHA ${ }^{3}$ \\ Jair Almeida CARNEIRO ${ }^{4}$ \\ Fernanda Marques da $\operatorname{COSTA}^{5}$
}

\begin{abstract}
${ }^{1}$ Estudante de Graduação em Enfermagem, Faculdades Unidas do Norte de Minas-Funorte, Rede Soebrás, email: carlagaguiar@yahoo.com.br

${ }^{2}$ Estudante de Graduação em Enfermagem, Faculdades Unidas do Norte de Minas-Funorte, Rede Soebrás, email: lindamfc@yahoo.com.br

${ }^{3}$ Enfermeira, Especialista em Saúde da Família, Docente da Universidade Estadual de Montes Claros-Unimontes e das Faculdades Unidas do Norte de Minas-Rede Soebrás, email: jucimerefdr@yahoo.com.br

${ }^{4}$ Médico, Mestre em Ciências da Saúde, Docente da Universidade Estadual de Montes Claros- Unimontes, email: jairjota@yahoo.com.br

5 Enfermeira, Mestre em Ciências da Saúde, Docente da Universidade Estadual de Montes Claros- Unimontes, email:fernandafjjf@yahoo.com.br
\end{abstract}

\section{Recebido em: 21/05/2014 - Aprovado em: 30/06/2014 - Disponibilizado em: 30/07/2014}

RESUMO: O estudo objetivou identificar os determinantes sociais, culturais e institucionais que interferem no acesso do homem aos serviços de atenção primária à saúde. Trata-se de um estudo descritivo de abordagem qualitativa. A coleta de dados foi realizada por meio da entrevista semi-estruturada, no período de maio a junho de 2013 na Estratégia de Saúde da Família de Montes Claros-MG. Os sujeitos do estudo foram dez homens que nunca procuraram os serviços da atenção primária. A análise dos dados foi baseada na técnica de análise do discurso. Os resultados encontrados demonstraram que as dificuldades vivenciadas pelos homens para a ausência ou baixa procura pelos serviços da atenção primária estão subdivididas em duas categorias: barreiras socioculturais e institucionais. Dentre as barreiras socioculturais destacam-se à construção da identidade de gênero, o entendimento sobre saúde, a crença inadequada sobre os serviços disponibilizados de saúde e a vergonha de expor o corpo a terceiros. Já as barreiras institucionais estão associadas à demora no atendimento, a horários inadequados e à inexistência de programas específicos para população masculina. Este estudo reforça a necessidade de se trabalhar a inserção do homem nas atividades da atenção primária a saúde.

Palavras-chave: Saúde do homem. Acesso aos serviços de saúde. Masculinidade. Atenção Primária à Saúde. Gênero e Saúde.

\section{SOCIOCULTURAL AND INSTITUTIONAL INTERFERENCE IN ACCESS TO HUMAN SERVICES PRIMARY HEALTH CARE}

\begin{abstract}
The study aimed to identify the social, cultural and institutional determinants that affect the man to primary care services to health access. This is a descriptive qualitative study. Data collection was conducted through semi - structured interviews in the period May-June 2013 in Family Montes Claros - MG Health Strategy. The subjects were ten men who never sought the services of primary care. Data analysis was based on the technique of discourse analysis. The results showed that the difficulties experienced by men to the absence or low demand for primary care services are divided into two categories: socio-cultural and institutional barriers. Among the sociocultural barriers include the construction of gender identity, the understanding of health, inadequate beliefs about the health services available and the shame of exposing the body to a third party. Have institutional barriers are associated with delay in treatment, the inappropriate times and the lack of specific programs for male population. This study reinforces the need to work the inclusion of man in the activities of primary healthcare.
\end{abstract}

Key words: Men's Health. Access to health services. Masculinity. Primary Health Care. Gender and Health.

\section{INTRODUÇÃO}

$\mathrm{O}$ interesse e as discussões acerca da relação

homens e saúde tem crescido ao longo dos anos, sobretudo aquelas direcionadas a temas como acesso e uso dos serviços disponíveis e perfis de morbimortalidade masculina. Um exemplo desta preocupação foi a Política

Nacional de Atenção Integral à Saúde do 
Homem, lançada em 2009 pelo Ministério da Saúde, com o objetivo de facilitar e ampliar o acesso da população masculina aos serviços de saúde, em resposta à observação de que os agravos do sexo masculino são um problema de saúde pública (COUTO et al., 2010).

O Brasil é o primeiro país da América Latina a implementar uma política nacional de atenção integral à saúde do Homem, o primeiro foi o Canadá. A partir dos princípios e diretrizes dessa política será possível orientar as ações e serviços de saúde para a população masculina, com integralidade e equidade, primando pela humanização da atenção (MEDEIROS; MENEZES; NAPOLEÃO, 2011).

$\mathrm{Na}$ atenção primária à saúde (APS) a demanda dos homens por atendimento é bastante inferior à das mulheres, devido a diferentes fatores, segundo os autores os homens geralmente buscam assistência em outros níveis de atenção. Essa busca por esses serviços de saúde se opõe ao preconizado pelo Ministério da Saúde, uma vez que a APS é considerada a porta de entrada e o principal contato dos usuários com os serviços de saúde independente de sexo ou idade (ALBANO; BASÍLIO; NEVES, 2010).

Entre as principais causas de morte masculina estão às doenças crônicas que podem ser prevenidas ou controladas por meio de intervenções em atitudes e práticas cotidianas que contribuem para a ocorrência desses problemas. Assim, esse estudo objetivou identificar os determinantes sociais, culturais e institucionais que interferem no acesso do homem aos serviços de APS. Acredita-se que o conhecimento das singularidades desse grupo específico, poderá possibilitar aproximação do serviço com a população masculina.

\section{ASPECTOS METODOLÓGICOS}

Trata-se de um estudo descritivo de abordagem qualitativa, que foi realizado na Estratégia de Saúde da Família (ESF) Delfino Magalhães na cidade de Montes Claros-MG, Brasil, no primeiro semestre de 2013. A escolha desta ESF baseou-se no tempo de implantação, característica considerada importante para a realização desse estudo. Essa ESF possui 13 anos de atuação junto a sua população. Atualmente a equipe atende 2.480 pessoas. Os homens cadastrados na faixa etária de 20 a 59 anos correspondem a $29,27 \%$ da população, perfazendo um total de 726 homens.

Os sujeitos do estudo foram homens com idade entre 25 e 59 anos, cadastrados na ESF que não procuraram os serviços disponíveis. Para identificação dos sujeitos foi realizado uma revisão nas fichas de cadastro das famílias (ficha A). Os homens selecionados participaram de uma entrevista individual semi-estruturada contendo algumas questões norteadoras.

Foram entrevistados dez homens selecionados aleatoriamente através de sorteio e para determinar o número de participantes foi empregado o critério de saturação de dados. Após transcrição e leitura dos relatos, os 
participantes foram codificados com nomes de metais para garantir o anonimato e o sigilo das informações. No tratamento dos depoimentos, utilizou-se o método de interpretação de sentidos, baseando-se em princípios hermenêutico-dialéticos que interpretam o contexto, as razões e as lógicas de falas, ações e inter-relações entre grupos e instituições (GOMES et al., 2005).

Inicialmente foi realizada a leitura compreensiva do material, em seguida procedeu-se a problematização das ideias e a busca de significados socioculturais e, por fim, estabeleceu-se um diálogo entre ideias problematizadas, informações provenientes de outros estudos acerca do assunto e o referencial teórico do estudo. A partir da análise e da interpretação dos depoimentos, foram agrupados os sentidos subjacentes em dois eixos de discussão: barreiras socioculturais e barreiras institucionais.

A coleta de dados ocorreu após aprovação do Comitê de Ética em Pesquisa da Sociedade Educativa do Brasil - SOEBRAS/ Faculdades Unidas do Norte de Minas - FUNORTE por meio do parecer $n^{\circ} 279.930$ de 05/2013 e caae: 14062713.2.0000.5141. Em todas as etapas da pesquisa seguiu-se o preconizado pela portaria 196/96 do Ministério da Saúde para a realização de pesquisa com seres humanos. Todos os homens que aceitaram participar do estudo autorizaram a gravação da entrevista assinando o Termo de Consentimento Livre e Esclarecido.

\section{RESULTADOS E DISCUSSÃO}

Os homens apesar de estarem inseridos em contextos sociais diferenciados têm uma percepção sobre a saúde parecida, eles não negam que possuem necessidades de saúde, porém relatam várias dificuldades em procurar os serviços oferecidos pela APS (SCHRAIBER et al., 2010). Dessa forma, os homens buscam menos os serviços de saúde se comparados às mulheres e quando o fazem geralmente a porta de entrada para o sistema de saúde não é feita pela APS (ALBANO; BASÍLIO; NEVES, 2010). Eles preferem procurar os serviços emergenciais em detrimento da ESF como se pode perceber nestes relatos nos quais os sujeitos afirmam nunca ter utilizado a ESF para consulta:

“Bem, eu acho que é ótimo né, pelo fato de tá principalmente próximo de nossa residência né, e apesar de que eu nunca procurei pra ter um pouco mais de conhecimento né [...]" (ZINCO).

"Muito bom apesar de eu não procurar [...]" (CHUMBO).

"Particularmente eu não conheço, quase não procuro [...]” (TITÂNIO).

"Muito bom, mais nunca procurei [...]" (FERRO).

"O atendimento de saúde aqui no bairro não, não, conheço não...” (AÇO).

“[...] eu não procurei, ainda.. eu nunca procurei o serviço de saúde pra mim [...]" (COBRE).

A ausência dos homens nesses serviços está relacionada a uma característica da identidade 
masculina referente ao seu processo de socialização. Nesse caso, essa identidade estaria associada à desvalorização do autocuidado e a preocupação incipiente com a saúde (SILVA, 2010).

Esse dado é encontrado em diversos estudos que comprovam a preferência dos homens por serviços voltados para o atendimento de problemas agudos (SCHRAIBER et al., 2010; MEDEIROS; MENEZES; NAPOLEÃO, 2011; COUTO et al., 2010). Esses estudos explicam a resistência masculina frente à APS, apontando diversas razões agrupadas, basicamente, em dois determinantes: barreiras socioculturais e institucionais.

\section{A Dificuldade de Acesso do Homem aos} Serviços de Atenção Primária: As

\section{Barreiras Socioculturais}

Para Couto et al. (2010) as dificuldades dos homens em procurar os serviços assistenciais podem estar relacionados a estrutura de identidade de gênero, a qual dificultaria a exposição de suas necessidades de saúde. Albano, Basílio e Neves (2010) ressaltam que essa resistência do homem em relação ao autocuidado está associada a uma cultura na qual os homens são educados como seres fortes e resistentes quando comparados às mulheres. Os depoimentos abaixo expressam esta situação:

"Acho, que deve ser pelo gênero mesmo né, masculino, de buscar atendimento quando ele percebe que não tem condições de locomover que a doença tá bem mais avançada" (COBRE).
“Às vezes também o homem se ocupa trabalhando, às vezes a mulher vai mais também porque elas têm mais coisa com saúde, e o homem encara tudo, é forte, né." (BRONZE).

"Olha [...] é porque o homem é mais desleixado, e mais orgulhoso [...] os homens fumam mais do que as mulheres, bebem mais e tem mais problemas respiratórios, mais problemas no fígado, mas eles nunca aceita [...]" (MERCÚRIO).

Com base nos discursos acima, vale destacar o achados de Gomes et al. (2011) que demostram que a organização do sistema de saúde, historicamente, vem sendo desenhada a partir da valorização do cuidado à saúde da mulher e da criança, tanto no meio profissional quanto no imaginário social e, assim, demarca as diferenças de abordagens de acolhimento por gênero, promovidas pelos serviços de saúde.

Corroborando com os dados apresentados, um estudo realizado por Figueiredo e Schraiber (2012) demonstra que a associação do cuidar está, realmente, na maioria das vezes, relacionada ao âmbito feminino. Os autores acreditam que na diferenciação de papéis por gênero presentes no imaginário social, principalmente masculino, entendem-se os cuidados de saúde como sendo característicos do gênero feminino.

As construções da masculinidade, por se estabelecerem contrárias ao universo feminino, se contrapõem também a comportamentos baseados no cuidado em 
saúde. Assim, os homens assumem maior dificuldade na busca por assistência em razão de sua autopercepção de necessidades de cuidados e pela noção de que esta é uma tarefa do universo feminino. Precisar ou procurar por cuidados de saúde é algo que desmerece sujeitos criados para assistir e prover. Essa imagem masculina do "ser forte" pode acarretar em práticas de pouco cuidado com o próprio corpo, tornando o homem vulnerável a uma série de situações (MACHIN et al., 2011).

Baasch (2007) afirma que os homens apresentam um entendimento de saúde como ausência de doença, entendimento este que se mostra claro na forma como eles utilizam os serviços de saúde o fazendo mediante demandas emergenciais e curativas. Gomes et al. (2011) também relatam em sua pesquisa que o homem só procura por atendimento de saúde com a intenção de tratar doenças e que pouco ou nada se interessam por ações preventivas, o que comprova uma tendência ainda predominante do modelo curativo no perfil de utilização dos serviços. Como foi evidenciado nos seguintes discursos:

"Só se eu tiver com muito sofrimento [...] Muito mal mesmo, aí eu vou pra consultar" (OURO).

“[...] só no ultimo caso, se não tiver jeito de... de sair, de a doença tá te deixando acamado caso contrário não procuro não [...]" (COBRE).

Simião (2010) afirma que ao homem caberia, então, ser forte - o que pode resultar em descuido com o próprio corpo. Pedir ajuda somente quando não suporta mais os desaba diante da doença já instalada e avançada.

Tendo em vista a assistência à saúde dos homens como um desafio para os serviços de atenção primária, Silva (2010) se posiciona dizendo que é bastante difundida a idéia de que os serviços desenvolvidos na APS são destinados, quase que exclusivamente, às mulheres, às crianças e aos idosos. Machim et al.( 2011) considera que esse fato afasta ainda mais os homens da unidade pois eles sentemse pouco à vontade nesse ambiente. Essa situação é evidenciada nos discursos abaixo: “[...] apesar de eu não procurar eu vejo pela minha mãe, meu pai que é mais idosos e faz tratamento lá” (CHUMBO).

“Bem eu suponho que o povo tem, deve ter um pouco de vergonha né, primeiramente $e$ pelo fato de sempre ter é mais mulheres nos PSF e posto de saúde" (ZINCO).

“[...] eu procuro mesmo hospital quando eu tô precisando mesmo [...] assim, normalmente graças a Deus foi poucas vezes, normalmente o PSF, é mais por causa dos meninos né, que tá doente mesmo, mas normalmente por causa deles" (TITÂNIO).

Outro fato que contribui para falta de cuidado dos homens com a sua saúde é a vergonha de ficar exposto diante do profissional de saúde. Para Gomes et al. (2011) essa justificativa, na visão dos homens, está associada a um sinal de fraqueza .

Possivelmente essa vergonha se associa à falta de hábito de se expor ao profissional de 
saúde. Ao contrário do homem, a mulher em sua socialização foi mais acostumada a ter o seu corpo exposto para a medicina. Essa divergência entre os gêneros, no que se refere ao olhar da medicina, pode ter contribuído para que a exposição da mulher seja vista com mais naturalidade do que a do homem (GOMES; NASCIMENTO; ARAÚJO, 2007).

Tal fato foi retratado nos seguintes depoimentos:

"[...] o homem fica com receio de procurar, por que geralmente a maioria são médicas né, não são homens então acho neste ponto eles tem um pouco mais de receio, e de vergonha” (ZINCO).

"Vergonha do profissional que tá lá, quando eu adoeço eu vou mesmo é na farmácia” (OURO).

“[...] o homem é mais orgulhoso [...] principalmente no que se refere aquele exame de próstata” (MERCÚRIO).

\section{A Dificuldade de Acesso do Homem aos} Serviços de Atenção Primária: As

\section{Barreiras Institucionais.}

Na pesquisa de Couto et al. (2010) destaca-se a inexistência de programas ou atividades voltados para a atenção aos homens, em particular os adultos jovens e em faixa reprodutiva, sendo estes atendidos em diversos programas que não são direcionados a essa população específica.

Esse também foi um dos motivos apontados pelos homens para a baixa demanda deles na APS neste estudo. Relatam que não procuram a unidade porque estas não disponibilizam e também não divulgam programas ou atividades direcionadas especificamente para eles. Esta situação foi descrita na seguinte fala:

"Acho que é a divulgação, eu acho que a divulgação pro lado das mulheres é bem mais $e$ nos homens acho que é [...] é que a assistência de saúde nos homens que fica a desejar"

Gomes, Nascimento e Araújo (2007) afirmam que os serviços de saúde da APS são considerados pouco aptos para absorver a demanda apresentada pelos homens, pelo fato de sua organização não contribuir para o acesso e as campanhas de saúde pública não atingirem este segmento populacional. De maneira geral o serviço está despreparado para o atendimento aos homens, mas também às mulheres.

Em sua maioria os homens procuram os serviços para serviços básicos, como vacinação, geralmente demandados por seus empregadores:

“eu já vacinei até mesmo porque o meu plano não cobre vacinas, mas eu nunca ouvi falar nos serviços direcionados para população masculina” (MERCÚRIO).

"vou ao PSF pra pegar remédio, vacinar e pegar receita” (CHUMBO).

Segundo Baasch (2007), o homem tem a percepção de que a organização do atendimento na APS voltado para uma agenda programada como sendo a causa da dificuldade para seu acesso ao serviço. De acordo com esta autora, os homens sentiriam 
mais dificuldades para serem atendidos, devido ao tempo perdido na espera da assistência como revela as enunciações:

“[...] ter que ir lá marcar, demora, tem que esperar, ocupa muito tempo né. Eu já fui, já há muito tempo atrás, já tentei [...] não consegui e desisti. O homem é sem paciência né [...] não tem paciência de ficar esperando, demora demais né [...] então é muito puxado para quem trabalha” (AÇO).

\section{“[...] chega lá tem aquela burocracia. Ah!!!} Tem que ficar esperando, tem aquela dificuldade toda, então normalmente agente não tem esse tempo todo pra esperar e acho que se marca aí é que demora demais" (TITÂNIO).

"[...] demora e o homem não tem muita paciência de esperar"(BRONZE).

Silva (2010) acrescenta que os homens preferem utilizar outros serviços de saúde, mais de pronto-atendimento, como farmácias e prontos-socorros, que responderiam mais objetivamente as suas demandas. Nesses lugares, eles são atendidos com mais rapidez e conseguem expor seus problemas com uma maior facilidade. Esta situação foi descrita na seguinte fala.

"[...] demora muito, aí eu vou na farmácia mesmo é mais rápido”(OURO).

"No hospital porque faz a ficha rapidinho e atende” (CHUMBO).

"Procuro o Pronto Atendimento Municipal Alpheu de Quadros, é um hospital bem maior e também tá bem mais rápido, pelo menos demora, mas sabe que vai ser atendido" (BRONZE).

O homem então pode ser identificado como alguém mais prático e objetivo. Isso interfere na adesão e na construção de vínculo com o serviço de saúde, na busca de serviços mais rápidos. No caso de tratamentos que requerem acompanhamento contínuo, isso é um fator importante que interfere na evasão (GOMES et al. 2011).

Segundo Simião (2010) outro fator impeditivo da procura dos homens pelos serviços de saúde refere-se à dificuldade de se encontrar postos de saúde ou ambulatórios abertos após as dezessete horas. Com o obstáculo da falta de tempo livre, restam a eles os serviços de urgência e emergência, visto que estes funcionam vinte e quatro horas por dia. Conforme demonstra o conjunto discursivo:

“Por causa do horário [...] eu trabalho o dia todo e as vezes que precisei foi nos horários que o posto tava fechado e também porque demora agenda lá pra frente” (AÇO).

"Porque os homens trabalha o dia todo, as mulheres tem mais tempo, se funcionasse de noite talvez os homens procuravam mais" (CHUMBO).

Neste ponto de vista, observa-se o fato de que as unidades básicas de atenção primária foram organizadas para o funcionamento em horários quase sempre incompatíveis com o "homem trabalhador", dando margens ao reconhecimento no mundo do trabalho de que apenas a mulher teria necessidades de uso regular dos serviços (GOMES et al. 2011). 
Ainda de acordo com Knauth, Couto e Figueiredo (2012) a ampliação do horário de funcionamento das unidades de saúde é vista como uma medida que pode atrair um maior número de usuários do sexo masculino. Isto pode ser constatado, por exemplo, em um dos serviços observados onde a ampliação do horário de atendimento trouxe melhorias na organização das atividades desenvolvidas, dentre as quais se destaca a ampliação do acesso da população masculina ao serviço.

Por isso, Couto et al. (2010) identificaram uma concentração maior de homens em unidades de saúde que criaram horários alternativos para o atendimento, sobretudo para os trabalhadores. Ressalta-se, nessas falas, que a demora em obter uma consulta, o tipo de marcação de horário, turnos de funcionamento e tempo de espera para ser atendido se constituem em princípios organizacionais que, possivelmente, impedem os homens desse estudo a priorizarem a APS como referência para atenção à saúde. Portanto, é possível inferir que, nesse caso, as dificuldades de acesso dos homens ao serviço de APS decorrem também da acessibilidade organizacional.

Esse achado coincide com os descritos por Lima et al. (2007) que identificaram que, apesar de existirem serviços de saúde próximos da casa do usuário, considerados de fácil acesso, sua escolha foi influenciada pela forma como foi recebido na unidade, pela confiança na experiência dos trabalhadores, o tipo de atendimento ofertado, a capacidade e a competência dos membros da equipe e a resolutividade desses serviços.

Silva (2010) acrescenta que o fato do processo de trabalho da ESF estar ainda focalizado no profissional médico e o potencial do profissional enfermeiro não estar sendo totalmente aproveitado leva a ESF a sustentar uma forma excludente de atendimento. Nesse serviço a prioridade é de quem chegar primeiro não se observando uma valorização dos problemas de saúde da população e o estímulo à autonomia do usuário. Sendo este fato considerado como uma das questões limitantes à consolidação do acolhimento, tendo em vista que o sucesso deste pressupõe agenda aberta e o desafio de conciliar o trabalho de assistência dentro da Unidade de Saúde com o trabalho externo.

A pouca procura masculina também aparece associada à ausência de acolhimento ou o acolhi- mento pouco atrativo, que pode estar relacionado à frágil qualificação profissional para lidar com o segmento masculino (GOMES et al., 2011).

Silva (2010) reforça ainda que o que contribuiria de forma positiva para melhora desse processo seria o estabelecimento de linhas guias e protocolos. Esses instrumentos poderiam nortear os procedimentos a serem adotados pela equipe, em especial pelos enfermeiros que, além de acolher poderiam contribuir para resolutividade das ações. Essa discussão remete a necessidade de meditar acerca da função que a ESF vem executando 
nos últimos anos, principalmente no contexto da promoção a saúde do homem.

\section{CONSIDERAÇÕES FINAIS}

Esta pesquisa reforça outros estudos nos quais os determinantes socioculturais e institucionais interferem no acesso do homem aos serviços da APS. Em relação às barreiras socioculturais a baixa demanda dos homens nos serviços da APS está associada à construção da identidade de gênero e da masculinidade. $\mathrm{O}$ homem é visto como forte e resistente e o cuidado com a saúde poderia demonstrar sinal de fraqueza o que, na visão deles, está relacionado ao âmbito feminino. Além disso, existe um entendimento de saúde equivocado, acreditam que só necessitam de

\section{REFERÊNCIAS}

ALBANO, Bruno Ramos; BASÍLIO, Márcio

Chaves; NEVES, Jussara Bôtto. Desafios para

a inclusão dos homens nos serviços de atenção primária à saúde. Revista

Enfermagem Integrada, Ipatinga: Unileste, v.3, n.2, p. 1-9. 2010.

\section{BAASCH, Mariani Santos. A percepção de}

homens da comunidade do alto aririú sobre seus cuidados em saúde. 2007. 57f. Trabalho de Conclusão de Curso (Graduação em Psicologia) - Universidade do Sul de Santa Catarina, Palhoça, 2007.

COUTO, Márcia Thereza et al. O homem na atenção primária à saúde: discutindo (in)visibilidade a partir da perspectiva de atendimento quando estão doentes e debilitados preferindo procurar pelos serviços emergenciais como farmácias e prontosocorro quando não conseguem mais lidar sozinhos com o problema e pouco se preocupam com atividades de prevenção e promoção da saúde.

O estudo oferece subsídios para se trabalhar à inserção do homem nas atividades desenvolvidas pela APS, para isso é necessário uma divulgação da existência de uma política voltada para esse segmento e que esta política seja verdadeiramente colocada em prática. A ampliação do horário de atendimento das unidades e a organização do atendimento deve ser uma possibilidade discutida entre profissionais e gestores.

gênero. Interface (Botucatu) [online], v. 14, n. 33, p. 257-270. 2010.

FIGUEIREDO, Wagner dos Santos;

SCHRAIBER, Lilia Blima. Concepções de gênero de homens usuários e profissionais de saúde de serviços de atenção primária e os possíveis impactos na saúde da população masculina. Ciência e saúde coletiva[online], v.16, suppl. 1, p. 935-944, 2012.

GOMES, Romeu; NASCIMENTO, Elaine Ferreira do; ARAÚJO, Fábio Carvalho de. Por que os homens buscam menos os serviços de saúde do que as mulheres? As explicações de homens com baixa escolaridade e homens com ensino superior. Cadernos de Saúde

Pública [online], v.23, n.3, p. 565-574, 2007. 
GOMES, Romeu et al. Os homens não vêm! Ausência e/ou invisibilidade masculina na atenção primária.Ciênc. saúde coletiva [online], v.16, suppl.1, p. 983-992. 201.1

GOMES, Romeu. Organização, processamento, análise e interpretação de dados: o desafio da triangulação. In: Minayo MCS, Assis SG, Souza ER, organizadores. Avaliação por triangulação de métodos: abordagem de programas sociais. Rio de Janeiro: Fiocruz; 2005. p. 185-221.

KNAUTH, Daniela Riva; COUTO, Márcia Thereza; FIGUEIREDO, Wagner dos Santos. A visão dos profissionais sobre a presença e as demandas dos homens nos serviços de saúde: perspectivas para a análise da implantação da Política Nacional de Atenção Integral à Saúde do Homem. Ciênc. saúde coletiva [online], v.17, n.10, p. 2617-2626. 2012.

LIMA, Maria Alice Dias da Silva et al.Acesso e acolhimento em unidades de saúde na visão dos usuários. Acta paul. enferm. [online], v.20, n.1, p. 12-17. 2007.

MACHIN, Rosana. Concepções de gênero, masculinidade e cuidados em saúde: estudo com profissionais de saúde da atenção primária. Ciênc. saúde coletiva [online], v.16, n.11, p. 4503-4512. 2011. MEDEIROS, Adriane Pinto de; MENEZES, Maria de Fátima Batalha de; NAPOLEÃO, Ana Maria Alves. Fatores de risco e medidas de prevenção do câncer de próstata: subsídios para a enfermagem. Revista Brasileira de Enfermagem [online], v. 64, n. 2, p.385-388. 2011.

SCHRAIBER, Lilia Blima. Necessidades de saúde e masculinidades: atenção primária no cuidado aos homens. Cadernos de Saúde Pública [online], v. 26, n. 5, p.961-970. 2010.

SILVA, Silvana de Oliveira. Cuidado na perspectiva dos homens: um olhar da enfermagem. 2010. 97 f. Dissertação (mestrado) - Universidade Federal de Santa Maria, Centro de Ciências da Saúde, Programa de Pós-Graduação em Enfermagem, Santa Maria, 2010.

SIMIÃO, Fernanda Cristina Nunes. Ele só chega nas últimas, quando não tem mais jeito: Atenção à sexualidade e à saúde reprodutiva dos homens nos discursos de profissionais do Programa Saúde da

Família em Recife. 2010.151f. Dissertação (mestrado) - Universidade Federal de Pernambuco. CFCH. Psicologia, Recife. 2010. 